\title{
Comments on Several FTF Annihilation Model Parameters
}

\author{
Julia Yarba, Fermilab \\ $26^{\text {th }}$ Geant4 Collaboration Meeting \\ (Hadronic Group) \\ Sept 15, 2021
}




\section{General Information (I)}

- Our attention to anti-baryons, among other reasons, is prompted by interest from Mu2e in modeling annihilation of anti-protons (FTF)

- For about a year there have been several (email) discussions that involved, at different times, a several people from Geant4 as well as interested users

- One of the recent points to discuss was production of pions in anti-proton annihilation on nuclei (Mu2e background study)

- We understand that there is interest in anti-protons from other experiments (AD experiments at CERN, astro-particle experiments,...), be it modeled by FTF or perhaps by an alternative model 


\section{Simplistic Overview of G4FTFAnnihilation (I)}

- Subject to correction by experts (Vladimir Uzh., sorry if there is any mistake here !)

- Modeling annihilation can be done via one of the following four scenarios:

- "3-shirt" diagram (three quark-antiquark strings)

- diquark-antidiquark string formation

- two quark-antiquark strings

- one quark-antiquark string

- The concept is the same for annihilation at rest or in flight although numerically there may be some differences

- Each scenario can be selected randomly according to the crosssection associated to it

- NOTE: Strings are subsequently fragmented but this is outside of the annihilation code 


\section{Simplistic Overview of G4FTFAnnihilation (II)}

- The cross-sections of the possible scenarios are defined through a collection of numeric parameters

- Such parameters, different sets for annihilation at rest or in flight, are currently hardcoded in G4FTFParameters class code, and duplicated within G4FTFAnnihilation

- There is one difference though: in G4FTFAnnihilation the cross-section for the diquark-antidiquark string formation is set to zero, thus the scenario is turned off, most likely because it's very CPU heavy.

- Varying the above mentioned parameters, and respectively the cross-sections, is likely to affect modeling of secondaries coming from the annihilation process; e.g. momentum spectra of secondary pions are likely to be sensitive. 


\section{Varying FTF/Annihilation Parameters by Hand}

Data: C. Amsler and F. Myher, Ann. Rev. Nucl. Part. Sci. 41, 219 (1991)

C.B. Dover et al., Prog. Part. Nucl. Phys., Vol.29, pp.87-173 (1992)

pbar annihilation on $\mathrm{H}$
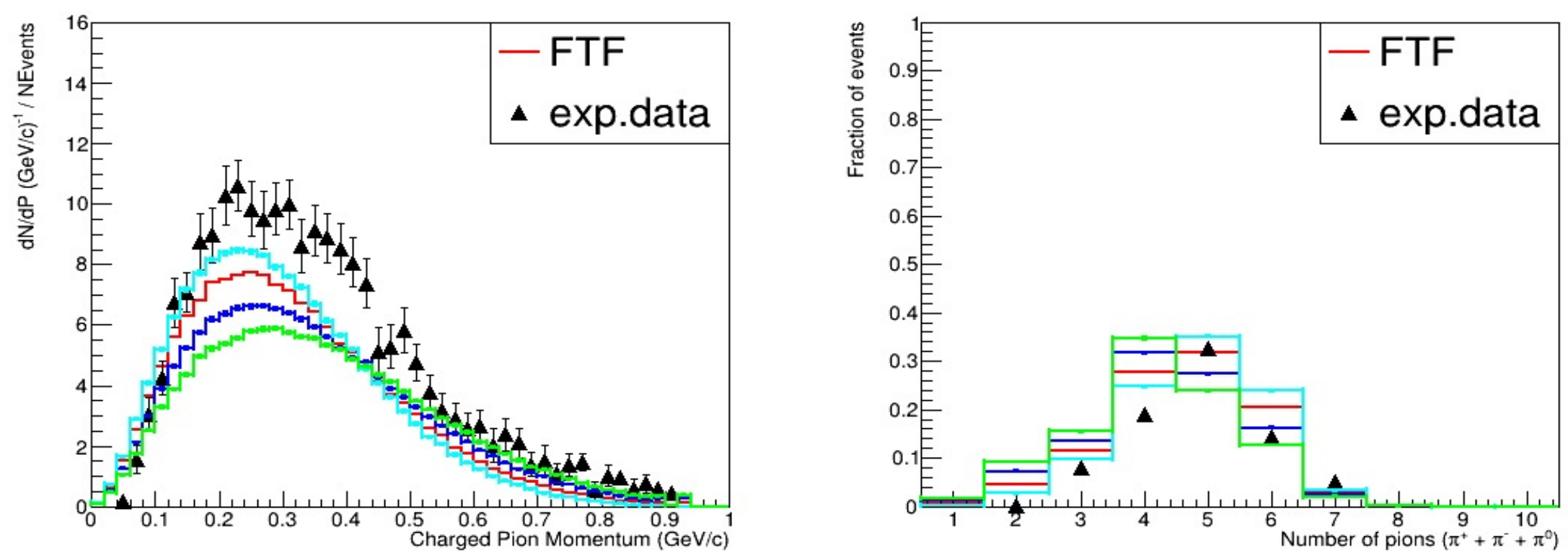

Increasing the probability of two quark-antiquark strings formation by a factor of 5 Turning OFF two quark-antiquark strings formation and one quark-antiquark string formation

Turning OFF "3-shirt" diagram and the probability of two quark-antiquark strings formation by a factor of 5 


\section{(Instead of) Summary}

- If agreed with FTF developers, G4FTFAnnihilation can be updated to pick up the parameters in question from G4FTFParameters; and some (or maybe all) of these parameters can be made configurable through G4FTFParameters and G4HadronicDeveloperParameters, for further exploring

- Unrelated to FTF annihilation model but still related to FTF handling interactions of antibaryons, one can also make configurable the switch that turns ON/OFF nuclear destruction process at energy below $2 \mathrm{GeV}$

- Of specific interest to Mu2e 